$\S=-1$

\title{
A Case Study on Solar Vapour Absorption Refrigeration System
}

\author{
Shabari Girish K.V.S., Praveen R., Dipesh Nair, Debjyoti Sahu* \\ Department of Mechanical Engineering, \\ Amrita School of Engineering, Bengaluru, Amrita Vishwa Vidyapeetham, India. \\ *Corresponding author E-mail: s_debjyoti@blr.amrita.edu
}

\begin{abstract}
Solar refrigeration may have applications in both developed and developing countries. Applications in developing countries such as airconditioning, vaccine storage or large scale food preservation have been the subject of scientific research. Ammonia-water based system has good COP but generally vapor absorption refrigeration system requires high generator temperature and high circulating ratio aided by a powerful pump. Our objective was to trivialize the role of circulating ratio and simultaneously achieve higher COP. A prototype model was designed, fabricated in laboratory using scrap material and tested; performance of the unit has been analyzed. A parabolic solar trough was used as a source of heat. Various components were modeled in computer and analyzed using ANSYS. The performances and effectiveness of the unit was studied by determining refrigeration effect (RE) and coefficient of performance (COP).
\end{abstract}

Keywords: Ammonia-water; CFD analysis; Parabolic solar collector; Vapor absorption.

\section{Introduction}

Solar refrigeration is used in areas with high insolation levels; where there is a demand for cooling and the grid power is expensive. Vapour absorption based refrigeration system uses process heat in a generator as main source of energy to run such system. Many industrial units generate thermal energy by burning fossil fuel whereas a few uses indirect heat from steam. After the process, heat is rejected to the surrounding as waste. This waste heat can be recovered and used in a heat operated refrigeration system, such as an absorption refrigeration cycle. In this study, the absorption of solar energy as the primary source of energy for an intermittent absorption refrigeration system is investigated. The energy emitted by the Sun in the form of heat can be harnessed easily using such technologies [1].

Klien et al. (2005), reported the sustainability of solar energy [2]. It mentions that refrigeration systems that use environmentfriendly refrigerants provide sustainability when compared to other refrigerant selections. Absorption refrigeration is the least intuitive of the solar refrigeration alternatives. Unlike the photovoltaic cell driven vapour compression system [3] and hybrid solar mechanical refrigeration options, the vapour absorption refrigeration system is considered a "heat driven" system that requires minimal mechanical power for the compression process [2]. It replaces the energy-intensive compression in a conventional vapour compression system with a heat activated "thermal compression system". Ammonia vapour exiting the evaporator is absorbed in a liquid solution of water-ammonia in the absorber [2]. The process is exothermic and cooling water is required to carry away the heat of absorption. The principle governing this phase of the operation is that, a vapour is more readily absorbed into a liquid solution when the temperature of the liquid solution is reduced. An overall system coefficient of performance (COP) can be estimated as the ratio of refrigeration capacity to input solar energy. Buehn et al. (2011), studied many aspects of solar ammonia absorption refrigeration, like the refrigerant-absorbent pairs, material compatibility and generation time [4]. Substantiating their work with market research, they reported all necessary properties of ammonia, essential for system analysis. Ammonia has zero ozone depletion potential and zero global warming potential thus far safe than any CFC, $\mathrm{HC}$ or HCFC based refrigerant [5]. Ammonia is a colourless gas, possessing a characteristic pungent smell and strongly alkaline; it is lighter than air, its specific gravity being 0.589 . It is easily liquefied and the liquid boils at $-33.7^{\circ} \mathrm{C}$. Ammonia gas has the power of combining with many substances. It is extremely soluble in water $\left(1: 1\right.$ at $\left.0^{\circ} \mathrm{C}\right)$. All the ammonia contained in an aqueous solution of the gas may be expelled by boiling. It does not support combustion, thus making it an ideal refrigerant in sea vessels until the CFC and HCFC became popular [5]. Montreal protocol being effective, CFC is banned and HCFC also needs to be eliminated at the earliest $[6,7]$.

Satish and Govind (2011), reported a detailed study of Vapor absorption system using Ammonia-water as refrigerant absorbent pair [8]. They introduced a rectifier to the conventional design to remove traces of water vapour in ammonia rich solution. However, crystalline salt usage can eliminate the use of a rectifier; especially salts with less volatility can be used. Some sand bed or perforated plates can also be used to increase the surface area of contact so that water vapour are condensed and are collected separately in liquid state. Thus separated ammonia vapour escape and complete the cycle. Refrigerants evaporate faster than other liquids because of their low boiling point. Cai et al., did a lumpedparameter dynamic model development for a absorption refrigeration cycle, thus design and operation parameters that affect the cycle performance are identified [9]. Decrease in Generator and condenser temperature increases the COP.

In present study the design, fabrication and testing of a small vapour absorption system is reported. The performances and effectiveness of the prototype as domestic refrigerator are analyzed. 


\section{Experimental Procedure}

\subsection{Design and Fabrication}

The Experiment is mainly conducted for calculating the COP (Coefficient of Performance) and RE (Refrigeration Effect) by observing and recording the temperature value of each component in the system (Fig. 1). The parabolic collector needs to be positioned in such a way that it is facing towards North and aligned to East-west direction [10]. The reflector is positioned in such a way that it is aligned to the median position of the sun's inclination. The experiment involves tracking the sun for every 60 minutes interval in both inclination and trajectory of the sun. This provides a maximum exposure of sunlight perpendicular to the mirrored surface. We recorded values of the generator temperature, condenser temperature and evaporator temperature. The complete sunlight received by the reflector is redirected towards the collector tube for heating the Ammonia-Water mixture in it. The heating of the Ammonia-Water mixture (assay 25\%, Nice Chemicals, Kochi) will lead to the evaporation of the ammonia and makes it to flow into the Dephlegmenter. Here the separation of Ammonia from water takes place as there is difference in their boiling points and state of being [8]. This also prevents any mixing of water and ammonia vapour. Water droplets pass through the bottom of Dephlegmator, and gets collected in the absorber after it passes through heat-exchanger. In this heat-exchanging unit, hot water transfers heat with cold ammonia and water mixture that is being pumped from the absorber. Ammonia being in gaseous state, it will pass into the condenser through the upper part of the Dephlegmenter.

A simple helical coiled copper tube placed inside a stainless steel container filled with water is used as water cooled condenser (In large setup water is sprinkled). Here, hot ammonia releases heat into water placed inside the condenser. So, the water is heated gradually and a temperature rise can be observed. Since the system pressure is high, the temperature of the surrounding water is enough to condense the refrigerant [2]. The condensed refrigerant will pass through expansion device, in order to decrease its pressure to certain value. Later, due to the gravitational force it flows down to the copper tubes that are arranged in particular shape which allows ammonia to stay long inside the Evaporator. The water in the absorber will pull the refrigerant ammonia from the evaporator which in turn absorbs the heat inside the evaporator this leads to Refrigeration Effect (RE). This effect basically depends on the quantity of ammonia entering the evaporator [4]. So, in order to enhance the refrigeration effect cold $\left(<0^{\circ} \mathrm{C}\right)$ concentrated ammonium hydroxide solution should be used so that more ammonia can be separated by the Dephlegmenter unit. In ideal situation ammonia gets mixed with water vapour as it enters the generator. To make this refrigeration cycle continuous a solution pump (small aquarium pump of capacity $4 \mathrm{~W}$ ) is used to pump the mixture from absorber to generator. This cycle continues throughout the day until there is enough sunlight to heat up the ammoniawater mixture.

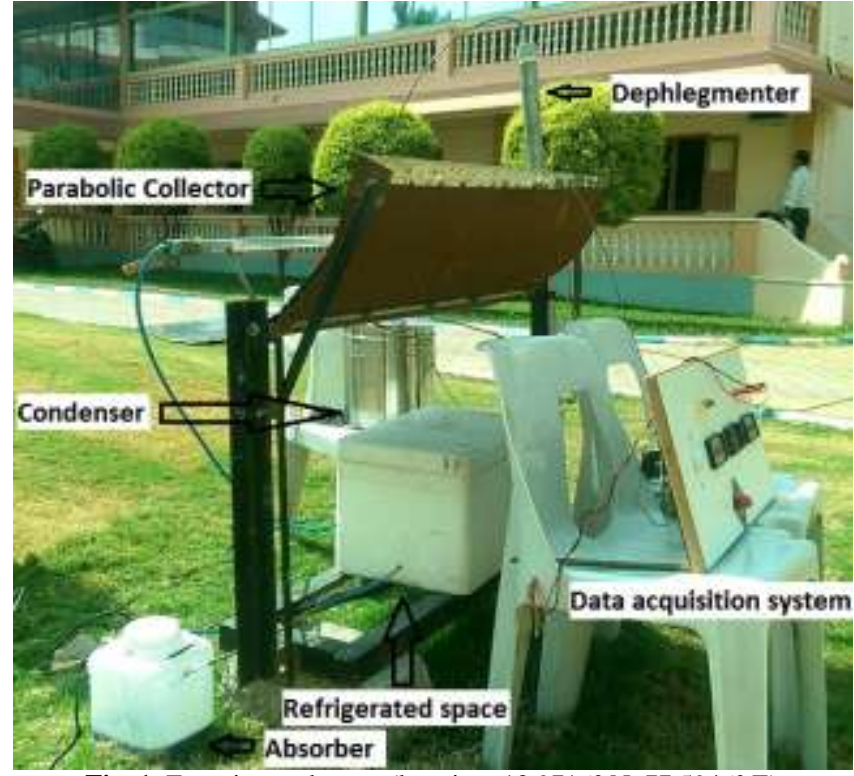

Fig. 1: Experimental setup (location: $12.9716^{\circ} \mathrm{N}, 77.5946^{\circ} \mathrm{E}$ )

Parameters for designing a parabolic trough [10]:

The equation of parabolic trough $\mathrm{y}=0.041677 \mathrm{x}^{2}$

(Eqn. 1)

Focal point $=1 /(4 \mathrm{a})$; where $\mathrm{a}=$ aperture $=0.41677 \mathrm{inch}$;

Therefore, focal point $=1 /(4 \times 0.41677)=6$ inch;

Reflector material: Stainless steel 304;

Total width of metal sheet: 24 inch;

Trough length: 49.16 inch;

Tracking facility is provided with the help of a nut sliding along a groove with a connecting rod from nut to the reflective surface.

Collector tube material: Double glazed evacuated tube (1 inch dia)

\subsection{Geometric Modeling}

As reported in the literature each component of this working model can be optimally designed so that the best performance is obtained from a given set of commercially available materials. Condenser can have various geometries and the expansion coil can have optimum number of pitch and coil diameter for a given length of copper tube. Therefore a CFD analysis of condenser coil and expansion coil is performed and reported in this work. Models of the expansion coil and condenser are prepared in the CATIA V5 (expansion coil geometry is shown in Fig.1) and later it is imported to ANSYS 15 workbench [11]. The model is processed further in the ANSYS workbench module to generate a structured mesh. The dimensions are specified as follows: Height $=40 \mathrm{~mm}$; Pitch $=8 \mathrm{~mm}$; Coil diameter $=100 \mathrm{~mm}$; Tube diameter $=3.176$ $\mathrm{mm}$.

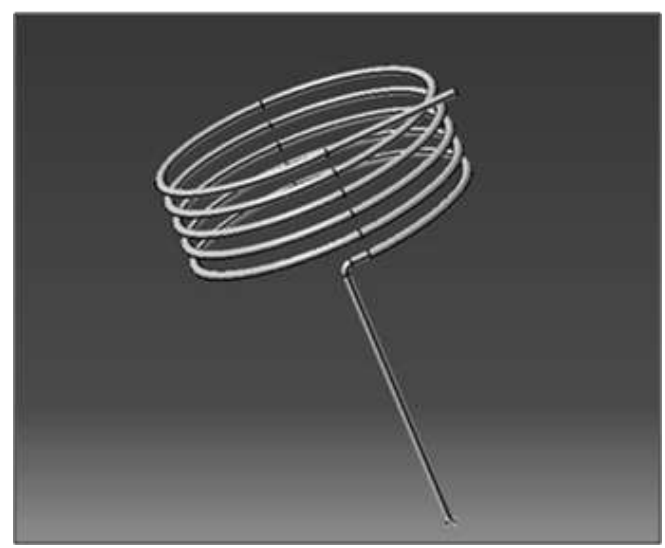

Fig.2: CAD Model of the Expansion Device 
The meshed model is processed for FLUENT setup where the analysis is done using density based solver under steady conditions. Boundary Conditions [12]: Pressure parameter is given for Inlet. Copper is taken as wall or tube material as it has high thermal conductivity (In industrial refrigeration, stainless steel is preferred for being non corrosive to ammonia). The inlet fluid is liquid Ammonia. Pressure inlet is $88 \mathrm{kPa}$, inlet temperature is $313 \mathrm{~K}$ and turbulence intensity $5 \%$ for $\mathrm{k}-\omega$ turbulence model.

\section{Results and Discussion}

\subsection{Analysis of the Condenser}

Computer simulations were carried out on three simple geometries to know the best shape of the condenser and the maximum temperature difference attained. As higher temperature drop in condenser generally signifies better rejection of heat to the surroundings [10].

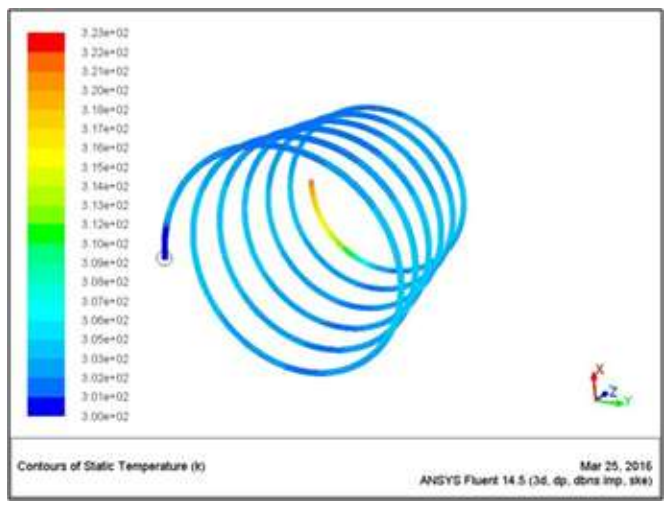

Fig.3: Helical Condenser

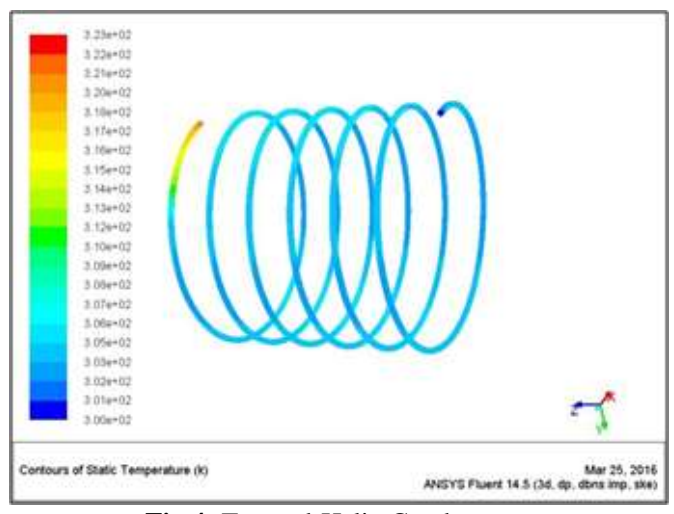

Fig.4: Tapered-Helix Condenser

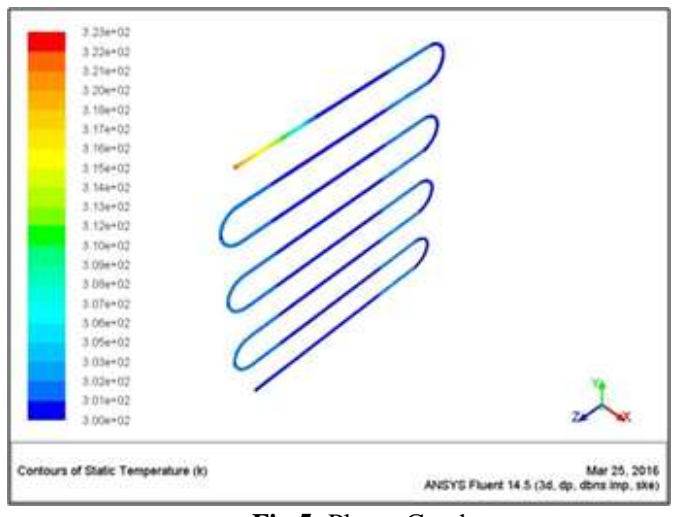

Fig.5: Planar Condenser
Table 1: Summary of results for different condenser geometry

\begin{tabular}{|l|l|l|l|}
\hline & Helical coil & Tapered-Helix & $\begin{array}{l}\text { Planar } \\
\text { Tube }\end{array}$ \\
\hline Inlet Temperature & $323 \mathrm{~K}$ & $323 \mathrm{~K}$ & $323 \mathrm{~K}$ \\
\hline Outlet Temperature & $301 \mathrm{~K}$ & $302 \mathrm{~K}$ & $304 \mathrm{~K}$ \\
\hline
\end{tabular}

From the temperature drop observed after simulation (Table 1);

helical shaped condenser coil (Fig. 3) was superior (temperature drop $>20 \mathrm{~K}$ ) over the other two shapes i.e. tapered helix coil (Fig.4) and planar condenser coil (Fig.5) considering a steady flow of mass through the similar length of tube.

\subsection{Analysis of Expansion Device}

Simulations help to determine the pressure drop observed in the expansion device (a capillary tube is the simplest option). The solution is initiated by considering the inlet as the reference zone [11]. Inlet and outlet boundary conditions/ parameters for the analysis and simulation are similar to Valladares et al. (2000) [12]. They reported a numerical simulation of a liquid overfeed system working with pure refrigerants under steady state condition. Their simulations were capable to predict the operating point of the system as function of the equipment's characteristics and the prevailing thermodynamic conditions. Pressures, temperatures, mass and volumetric flow rates were computed at all relevant points.

Table 2: Expansion coil parameters

\begin{tabular}{|c|c|c|}
\hline & INLET & OUTLET \\
\hline Pressure $(\mathrm{kPa})$ & 88 & 3.45 \\
\hline Temperature $(\mathrm{K})$ & 313 & 305 \\
\hline Total Heat Transfer Rate $(\mathrm{kW})$ & \multicolumn{2}{|c|}{20} \\
\hline Mass Flow Rate $(\mathrm{kg} / \mathrm{s})$ & \multicolumn{2}{|c|}{} \\
\hline
\end{tabular}

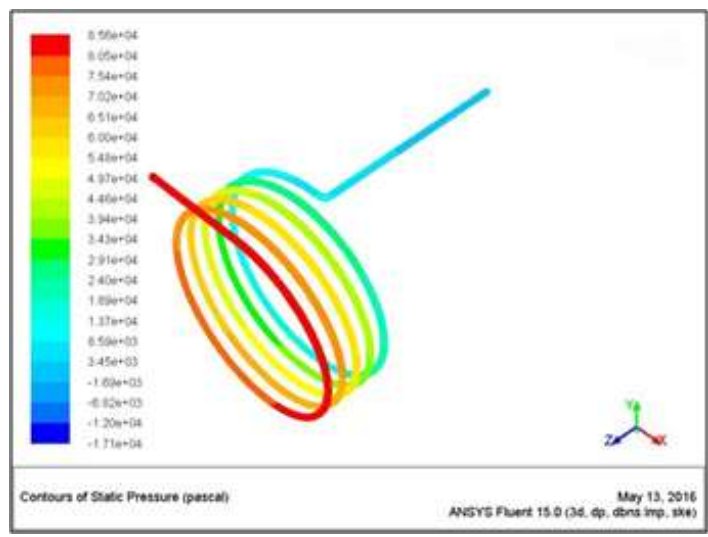

Fig.6: Pressure Contour in Expansion Device

The solution converged after completing1500 iterations in our laboratory. From the pressure contour (Fig.6), we observe that there is a major pressure drop which facilitates the cooling effect. Magnitude of pressure drop and heat transfer rate thus computed is given in Table 2 . A pressure drop of $\sim 85 \mathrm{kPa}$ is associated with a temperature drop of $\sim 8 \mathrm{~K}$ when the mass flow rate is $0.0132 \mathrm{~kg} / \mathrm{s}$. Therefore a higher mass flow rate will cause even better heat transfer.

\subsection{Experimental Results}

During this test the sun was tracked on a 60 -minute basis. The collector was able to concentrate sunlight available and use it to sustain a reasonable temperature in the collector tube [10]. After initial heating, maximum temperature of the collector is recorded between 10:30 am and 4:30 pm. The experiment readings were recorded at approximately 10:30 am onwards. The generator was aligned north-south and facing west, it was then tilted to align with a median point of the inclination between the start time and the midday point. Temperature of the collector tube, condenser, and evaporator were recorded using thermocouple and calibrated with the help of a glass thermometer. Series of readings are taken 
for a week and tabulated. Solar flux [1] readings are noted with the help of a flux meter along with the temperatures. It helps in determining the amount of solar insolation available at our location $\left(12.9716^{\circ} \mathrm{N}, 77.5946^{\circ} \mathrm{E}\right)$. Pump is started 60-90 minutes before the first reading is taken, as Generator takes time for heating. Because of temperature difference in the collector and the absorber, a drop in pressure level occurs which drives the refrigerant back to the absorber. Evaporator was observed to decrease the temperature, compared to the surroundings [13].

Neglecting the pump work COP can be approximated using the formula:

$$
\frac{[(\mathrm{Tg}-\mathrm{Tc}) \times \mathrm{Te}]}{[(\mathrm{Ta}-\mathrm{Te}) \times \mathrm{Tg}]}=C O P
$$

In our system, generator temperature $(\mathrm{Tg})$ is the highest followed by condenser temperature (Tc) is above the atmospheric temperature $(\sim 310 \mathrm{~K})$ whereas absorber temperature $(\mathrm{Ta})$, is near to the atmospheric temperature $\sim 308 \mathrm{~K}$. Temperature inside the refrigerated space was found to be near $\sim 296-298 \mathrm{~K}$ during 2:30 pm and 4:30 pm which seems promising considering the atmospheric temperature to be $>308 \mathrm{~K}$. COP values which are given in Table 4 are calculated after taking readings from the field. During morning (till 11:00 am) each day the temperature inside the refrigerated space is high hence COP also expected to be high. Coefficient of performance depends on the temperature difference of the components of system [13]. From the Eqn. (2), actual COP has been calculated from the recorded field temperatures. COP values are tabulated with respect to time of the day (Table 3). Graph is plotted based on the average generator temperature which gives more insight (Fig. 9). A fluctuation is visible in performance. Micallef and Micallef [2010] reported that a vapour absorption refrigeration system with solar collector may be susceptible to temperature fluctuation throughout the day hence may give an inconsistent performance [14]. However, a hybrid system with dual heating facility may give a continuous as well as consistent COP.

Table 3: COP calculation from recorded temperature on field

\begin{tabular}{|l|r|r|r|r|}
\hline DATE & $10: 30 \mathrm{AM}$ & \multicolumn{1}{|c|}{$12: 30 \mathrm{PM}$} & $2: 30 \mathrm{PM}$ & $4: 30 \mathrm{PM}$ \\
\hline $09-05-2016$ & 0.94 & 0.97 & 0.79 & 0.57 \\
\hline $10-05-2016$ & 0.97 & 0.81 & 0.63 & 0.5 \\
\hline $11-05-2016$ & 0.78 & 0.98 & 0.42 & 0.62 \\
\hline $12-05-2016$ & 0.95 & 0.94 & 0.81 & 0.59 \\
\hline $13-05-2016$ & 0.97 & 0.94 & 0.83 & 0.97 \\
\hline $14-05-2016$ & 0.8 & 0.89 & 0.87 & 0.63 \\
\hline $15-05-2016$ & 0.82 & 0.92 & 0.57 & 0.47 \\
\hline
\end{tabular}

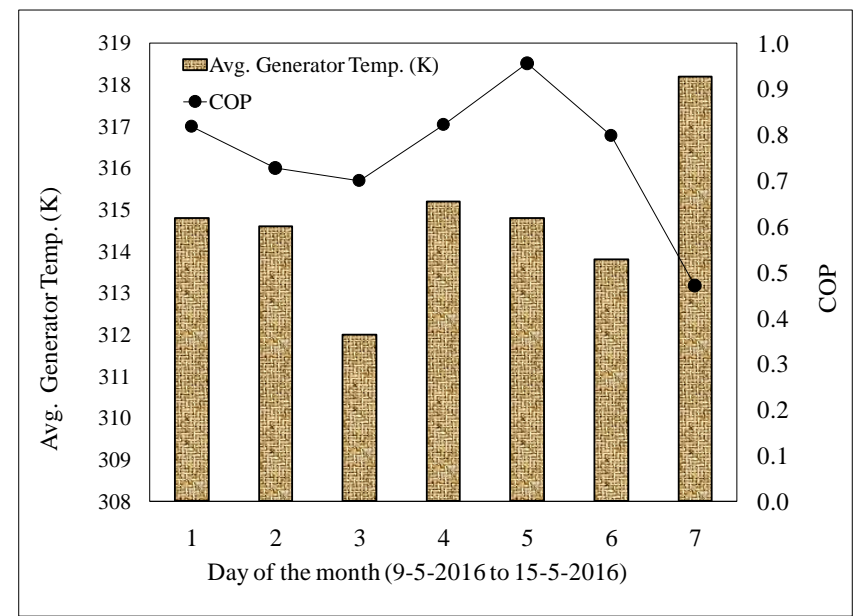

Fig. 9: Variation of COP with respect to the generator temperature

\section{Conclusion}

To present a test case we selected a solar powered vapour absorption refrigeration system with Ammonia-water as refrigerant.
Simulations were performed on the important components and different parameters were studied. The pressure and temperature contour obtained from the analysis may help in deciding the condenser geometry and pitch of the expansion coil. Helical coil condenser geometry was selected considering higher temperature gradient. The set up was then modified and temperatures of different components were noted. Performance analysis graphs were plotted vis-a-vis the COP. The results obtained in the experiment were quite promising for the composition of the refrigerant used and the collector capacity. The system is simple and economical. We could observe cooling effect in the refrigerated space throughout the day. With evacuated tubes connected to the parabolic collector that affect a higher generator temperature, the refrigeration effect might improve in the modified setup in future.

\section{References}

[1] Sukatme SP, Solar Energy, Tata McGraw-Hill, New Delhi, 2004. p. 37-68.

[2] Klien D, Reindl T, Solar Refrigeration, ASHRAE Journal, 2005;47 (9), 526-530.

[3] Ewert M.K, Bergeron DJ, Foster RE, Lafleur O, Solar 2002, Am. Sol. Energ. Soc., Reno, Nevada, 2000.

[4] Buehn J, Hudspeth A, Villanueva G, ISAAC Solar Ice Maker. Welcome to Energy Concepts. 2011. http://www.energyconcepts.com/isaac

[5] Velders GJM, Andersen SO, Daniel JS, Fahey DW, McFarland M, The importance of the Montreal Protocol in protecting climate, Proceedings of National Academy of Science of the USA, 2007;104 (12) 4814-4819.

[6] Abinav R, Gautham N, Sahu D, A case study of a low power vapour compression refrigeration system, IConAMMA 2016, Bengaluru, India, 2016. http://iopscience.iop.org/article/10.1088/1757899X/149/1/012226

[7] Dinesh B, Sai M, Dishal KT, Sahu D, A case study of a low power vapour adsorption refrigeration system, IConAMMA 2016, Bengaluru, India, 2016. http://iopscience.iop.org/article/10.1088/1757899X/149/1/012227

[8] Satish R, Govind M, Analysis of Ammonia-Water Vapour Absorption Refrigeration System based on First Law of Thermodynamics Int. J. Scientific Eng. Research, 2011; 2(8), 1-7.

[9] Cai W, Sen M, Paolucci S, Dynamic simulation of an ammoniawater absorption refrigeration system, University of Notre Dame, 2010. https://www3.nd.edu/ msen/Research/Preprints/Cai7.pdf

[10] Cabrera FJ, Garcia AF, Silva RMP, Garcia MP, 2013 Use of Parabolic trough solar collectors for solar refrigeration and airconditioning applications, Review Article, Renewable and Sustainable Energy Reviews, 2010; 103-118.

[11] ANSYS Fluent user Guide, release 14.5, Nov 2012 and ANSYS fluent theory guide 12.0.

[12] Valladares O, Segarra CD, Oliet C, Danov S, Numerical Studies of Refrigerating Liquid Overfeed Systems Working With Ammonia and R-134a, Centre Tecnologic de Transferencia de Calor (CTTC) Laboratori de Termotecniai Energetica; Universitat Politecnica de Catalunya, 2000; 327-334.

[13] Rathore MM, Thermal Engineering, Tata McGraw-Hill, 3rd edition, New Delhi, 2008. p. 447-468.

[14] Micallef D, Micalef C, Mathematical model of a vapour absorption refrigeration unit, Int. J. Simul. Model 2010;9(2), 86-97. 